\title{
CARACTERIZAÇÃO E BIODIGESTÃO ANAERÓBIA DOS DEJETOS DE CAPRINOS ${ }^{1}$
}

\section{ANA C. A. ORRICO ${ }^{2}$, JORGE DE LUCAS JÚNIOR ${ }^{3}$, MARCO A. P. ORRICO JÚNIOR ${ }^{4}$}

RESUMO: Quantificar e caracterizar os dejetos gerados por cabras Saanen em quatro categorias de idade e alimentadas com três dietas e na seqüência, e promover a biodigestão anaeróbia dos dejetos constituíram os objetivos deste trabalho. Para a produção de dejetos, foram utilizadas 36 cabras Saanen, com idades entre 2 e 4 (C1), 4 e 8 (C2), 8 e 12 (C3) e acima de 12 meses (C4), alimentadas com as dietas 1 (D1: 80\% volumoso (Vol) e 20\% concentrado (Con)); 2 (D2: 60\% Vol e $40 \%$ Con) e 3 (D3: 40\% Vol e 20\% Con). Foram quantificadas as produções diárias de fezes e urina e seus teores em N, P, K, Ca, Mg, Na, Fe, Cu, Zn e Mn. Com a mistura das fezes e urina de todas as categorias, separadas segundo as dietas, foram abastecidos biodigestores batelada, com capacidade para $4 \mathrm{~L}$ de substrato em fermentação. A C1 apresentou menor $(\mathrm{P}<0,05)$ excreção de fezes (164,1 g de MS (massa seca)/animal por dia) e o menor consumo de alimento (362,2g MS (massa seca)/animal por dia). As maiores concentrações de $\mathrm{N}, \mathrm{P}, \mathrm{K}, \mathrm{Ca}, \mathrm{Mg}$ e K ocorreram nas fezes e urina geradas por cabras da $\mathrm{C} 4$ e alimentadas pela D3. O substrato preparado com dejetos oriundos da D3 apresentou $45 \%$ de redução nos teores de sólidos voláteis (SV). Os substratos preparados com dejetos obtidos de animais alimentados com a D3 produziram mais biogás $(\mathrm{P}<0,01)$ por kg de sólidos totais e SV adicionados.

PALAVRAS-CHAVE: biogás, sólidos totais, sólidos voláteis.

\section{CHARACTERIZATION AND ANAEROBIC DIGESTION OF GOAT MANURE}

ABSTRACT: This study aimed quantify and characterize the manure generated by Saanen goats in four categories of age, fed with three diets and then promote the anaerobic digestion of the manures. Thirty six goats were used with ages between 2 and 4 (C1), 4 and 8 (C2), 8 and 12 (C3) and above 12 months (C4), fed with the diets 1 (D1: 80\% forage (Fo) and 20\% concentrated (Co)), 2 (D2: 60\% Fo and 40\% Co) and 3 (D3: 40\% Fo and 20\% Co). The daily productions of feces and urine and its contents of $\mathrm{N}, \mathrm{P}, \mathrm{K}, \mathrm{Ca}, \mathrm{Mg}, \mathrm{Na}, \mathrm{Fe}, \mathrm{Cu}, \mathrm{Zn}$ and $\mathrm{Mn}$ were quantified. A mixture of feces and urine of all the categories of age was used; separated according to the diets, for the supply of batch digesters, with capacity of 4.0 liters of substrate in fermentation. The $\mathrm{C} 1$ presented smaller excretion $(\mathrm{P}<0.05)$ of feces $(164.1 \mathrm{~g}$ of MS/animal per day) and the smallest food consumption (362.16 g MS/animal per day). The largest concentrations of $\mathrm{N}, \mathrm{P}, \mathrm{K}, \mathrm{Ca}, \mathrm{Mg}$ and $\mathrm{K}$ occurred in the feces and urine generated by goats of $\mathrm{C} 4$ and fed by D3. The prepared substrate with manures originating from of D3 presented $45 \%$ of reduction in the contents of volatile solids (VS). The prepared substrate with manures of animals fed with D3 produced more biogas $(\mathrm{P}<0.01)$ for $\mathrm{kg}$ of total solids and VS added.

KEYWORDS: biogas, total solids, volatile solids.

\footnotetext{
${ }^{1}$ Projeto financiado pela FAPESP. Extraído da dissertação de Mestrado da primeira autora.

${ }^{2}$ Zootecnista, Profa. Dra., Universidade Católica Dom Bosco, Campo Grande - MS, zoo@ ucdb.br

${ }^{3}$ Eng $^{\mathrm{o}}$ Agrônomo, Prof. Titular, Departamento de Engenharia Rural, UNESP-Jaboticabal - SP, jlucas@ fcav.unesp.br

${ }^{4}$ Zootecnista, Mestrando pelo Programa de Zootecnia da UNESP - Jaboticabal, Departamento de Engenharia Rural, Jaboticabal SP, Fone: (0XX16) 3209-2638, marcoorrico@yahoo.com.br. Bolsista da FAPESP.

Recebido pelo Conselho Editorial em: 13-7-2006
}

Aprovado pelo Conselho Editorial em: 28-6-2007 


\section{INTRODUÇÃO}

Aproximadamente $94 \%$ do rebanho caprino brasileiro está na região Nordeste, onde prevalecem condições edafoclimáticas desfavoráveis. Nessa situação, os caprinos assumem grande importância social, pois chegam a ser a única fonte de renda em determinadas circunstâncias e deles depende a sobrevivência de muitos nordestinos (RIBEIRO \& RIBEIRO, 2001). DUBEUF et al. (2004) ressaltaram a grande importância dos caprinos no desenvolvimento de zonas rurais.

A produção de pequenos ruminantes como fonte de alimentos protéicos, associada a técnicas de tratamento e reciclagem dos resíduos gerados na atividade, representa alternativa viável em termos ambientais e econômicos, sobretudo para pequenos produtores ou em áreas reduzidas. Para EL AICH \& WATERHOUSE (1999), a criação de ovinos e caprinos justifica-se pela elevada capacidade de adaptação desses animais, sobretudo dos caprinos, em locais como regiões de estepe, de rochas, declives ou mesmo consideradas pobres e, portanto, desprezadas para agricultura.

Uma estratégia para o desenvolvimento de determinada atividade, sem que haja maior comprometimento das reservas energéticas e do ambiente, ocasionados pela disposição inadequada dos resíduos gerados nos sistemas produtivos, é adotar sistemas para a reciclagem desses resíduos, promovendo-se, dessa forma, a geração de energia, utilizando-se de fontes renováveis (AMORIM et al., 2004).

Os resíduos de origem animal constituem-se em importante parcela da biomassa, e sua utilização em sistemas de reciclagem mostra-se favorável sob aspectos econômicos e ambientais. JARDIM (1977) estimou que uma cabra adulta produzisse $600 \mathrm{~kg}$ de dejetos ao ano, em média. Ao associar-se a quantidade produzida com a qualidade desse material, que, segundo AGRAZ (1989), pode conter até 3 e $2 \%$ de $\mathrm{N}$ e $\mathrm{P}$, respectivamente, ter-se-ia um excelente substrato para a digestão anaeróbia, pois, além de as concentrações de nutrientes otimizarem a atividade dos microrganismos, poderiam retornar ao solo de maneira não-prejudicial e mais assimilável pelas plantas.

A digestão anaeróbia é um dos vários processos existentes para tratamento dos resíduos e representa um método bastante atrativo, pois promove a geração do biogás, como fonte de energia alternativa, e do biofertilizante (AUGENSTEIN et al., 1994 e SAHA, 1994). Além disso, a prática contribui para o saneamento, reduzindo o número de patógenos no produto final.

AL-MASRI (2001) avaliou o desempenho de biodigestores anaeróbios abastecidos com dejetos de caprinos e ovinos. Para tanto, utilizou biodigestores modelo batelada, mantendo os substratos retidos por 40 dias e em banho-maria, na temperatura de $30{ }^{\circ} \mathrm{C}$. A redução de sólidos totais e voláteis (ST e SV) foi de 72,6 e 58,2\%, respectivamente, quando se utilizaram como substrato dejetos de ovinos e 71,3 e $58,1 \%$ em biodigestores abastecidos com dejetos de caprinos. MISI \& FORSTER (2001) avaliaram biodigestores abastecidos com misturas à base de dejetos de caprinos, ovinos e bovinos, resíduos do processamento de frutas e cama de frangos, em diferentes proporções. Quando os dejetos de ovinos e caprinos perfizeram 70\% da mistura, obtiveram-se como valores: produção total de 4.189,50 $\mathrm{mL}$ de $\mathrm{CH}_{4}$ (em 35 dias de retenção e em biodigestores com capacidade de $1.000 \mathrm{~mL}$ ); $0,14 \mathrm{~m}^{3}$ de metano/kg de $\mathrm{SV}$ adicionado, $0,42 \mathrm{~m}^{3} \mathrm{de} \mathrm{CH}_{4} / \mathrm{kg}$ de SV reduzido, e $34,1 \%$ de redução de $\mathrm{SV}$.

Com base na relevância da reciclagem dos resíduos gerados na caprinocultura ao desenvolver-se este trabalho, teve-se o objetivo de avaliar as prováveis interferências das dietas sobre a quantidade e qualidade dos dejetos excretados por cabras em diferentes idades e avaliar os efeitos dessas variáveis sobre a biodigestão anaeróbia dos dejetos.

\section{MATERIAL E MÉTODOS}

Para a produção dos dejetos, foram utilizadas 36 cabras da raça Saanen, distribuídas em quatro faixas etárias ( 2 a 4; 4 a 8; 8 a 12 e acima de 12 meses) e alimentadas com três dietas: dieta 1 ( $80 \%$ volumoso e $20 \%$ concentrado), dieta 2 (60\% volumoso e $40 \%$ concentrado) e dieta 3 (40\% volumoso e $20 \%$ concentrado). O volumoso foi o feno de Tifton 85 (Cynodon dactylon), e o 
concentrado foi composto por $46,8 \%$ de milho moído, $4 \%$ de soja em grão, $11 \%$ de farelo de soja, 9,1\% de farelo de algodão, 5,2\% de farelo de trigo, 17,9\% de feno de alfafa, $5 \%$ de núcleo leite e $1 \%$ de calcário. Para o cálculo de oferta do volumoso, contabilizou-se o feno de alfafa contido no concentrado. As dietas foram ajustadas para atender às exigências dos animais, segundo recomendações do NRC (1981).

Para a colheita dos dejetos, as cabras foram alojadas em gaiolas metabólicas individuais de 0,7 x 1,4 m (L x C), contendo comedouros e bebedouros acoplados, assim como coletor de dejetos, possibilitando a colheita de fezes e urina separadamente. A alimentação foi fornecida ad libitum, em duas refeições diárias, havendo à disposição dos animais água e sal mineral. Após a adaptação dos animais às dietas e instalações por 14 dias, os dejetos foram coletados por cinco dias consecutivos, diariamente.

\section{Caracterização quantitativa e qualitativa dos dejetos}

As amostras de fezes foram pré-secas a $55^{\circ} \mathrm{C}$ e moídas em moinho de facas para serem utilizadas na determinação dos sólidos totais (ST) e voláteis (SV), e também empregadas para a digestão sulfúrica e conseqüente quantificação de $\mathrm{N}, \mathrm{P}, \mathrm{K}, \mathrm{Ca}, \mathrm{Mg}, \mathrm{Na}, \mathrm{Fe}, \mathrm{Cu}, \mathrm{Zn}$ e $\mathrm{Mn}$. A urina foi digerida in natura e com o extrato determinado os elementos mencionados.

\section{Biodigestão Anaeróbia}

Para a composição dos substratos de abastecimento dos biodigestores, foram utilizados os dejetos excretados por todos os animais que recebiam a mesma dieta, independentemente da categoria de idade, utilizando-se de quatro biodigestores para cada dieta avaliada. Para o abastecimento dos biodigestores, coletaram-se os dejetos produzidos em $24 \mathrm{~h}$, a fim de evitar fermentação excessiva e perdas na geração de biogás. Os substratos foram preparados utilizando-se de água para a diluição e adotando-se teor de ST em torno de 8\% (LUCAS JÚNIOR, 1994), sendo a mistura homogeneizada em liquidificador industrial. Foram utilizados biodigestores batelada de bancada, constituídos por dois vidros, cada um com capacidade útil para substrato em fermentação de $2 \mathrm{~L}$, fechados por tampa de borracha, com um tubo metálico acoplado no centro para permitir a saída do biogás produzido. Os vidros foram interligados, com tubos de látex, a gasômetros metálicos com "selo d'água", permitindo o armazenamento e a medida do biogás produzido. Os biodigestores foram avaliados por todo o tempo em que apresentaram produção de biogás, não se estabelecendo tempo de retenção.

Para a determinação de ST e SV, utilizou-se de metodologias descritas por APHA (1995). A digestão sulfúrica para a quantificação dos nutrientes foi realizada em digestor Digesdahl, da Hach. Com o extrato resultante, foi possível quantificar os teores de N (destilador Micro - Kjeldahl), P, K, $\mathrm{Ca}, \mathrm{Mg}, \mathrm{Na}, \mathrm{Fe}, \mathrm{Cu}, \mathrm{Zn}$ e Mn, em espectrofotômetro de absorção atômica, modelo GBC 932 AA.

No dia do abastecimento dos biodigestores, os dejetos apresentaram teores de ST iguais a $30,8 \%$ para animais alimentados com a dieta $1 ; 25,3 \%$ para os que consumiram a dieta 2 , e $23,3 \%$ para os que ingeriram a dieta 3 , dos quais $87,8 \% ; 85,7 \%$ e $87,5 \%$ eram voláteis, respectivamente.

Os volumes de biogás produzidos foram determinados medindo-se o deslocamento vertical dos gasômetros e multiplicando-se pela área da seção transversal interna dos gasômetros, ou seja, $0,03696 \mathrm{~m}^{2}$. Após cada leitura, os gasômetros foram zerados, utilizando-se do registro de descarga do biogás. A correção do volume de biogás para as condições de 1 atm e $20{ }^{\circ} \mathrm{C}$ foi efetuada com base no trabalho de CAETANO (1985). Os potenciais de produção de biogás foram calculados utilizando-se dos dados de produção diária e das quantidades de dejetos in natura de substrato, de ST e de SV adicionados nos biodigestores, além das quantidades de SV reduzidas durante o processo de biodigestão anaeróbia. Os valores foram expressos $\mathrm{em}^{3}$ de biogás por $\mathrm{kg}$ de substrato, de dejeto ou de ST e SV.

Os resultados gerados no ensaio de caracterização dos dejetos foram comparados pelo teste de Tukey, a 5\% de probabilidade, adotando-se o delineamento inteiramente casualizado, em esquema fatorial $3 \times 4$ (dietas x categorias), considerando-se cinco dias de coleta e três animais por 
tratamento. Para avaliação do efeito das dietas na produção de biogás, adotou-se o delineamento inteiramente casualizado, sendo composto de três tratamentos (dietas), com quatro repetições (biodigestores), sendo as médias comparadas pelo teste de Tukey, a $5 \%$ de probabilidade. Para a comparação das médias das variáveis, utilizou-se do SAS (1990).

\section{RESULTADOS E DISCUSSÃO}

A categoria 1 apresentou menor $(\mathrm{P}<0,05)$ excreção de fezes $(164,1 \mathrm{~g}$ de MS/animal por dia) do que as demais $(247,6 ; 272,6$ e 298,3g de MS (massa seca) de fezes/animal por dia, nas categorias 2; 3 e 4, respectivamente). Provavelmente, essa ocorrência esteja associada ao menor $(\mathrm{P}<0,05)$ consumo apresentado pelos animais da categoria 1 (362,2 g MS/animal por dia) em relação aos animais das demais categorias $(749,7 ; 742,2$ e 820,9 g de MS/animal por dia, nas categorias 2; 3 e 4). A melhor qualidade da dieta 3 fica evidenciada ao analisarem-se os valores de consumo de alimento e produção de fezes (760,3 e 251,9g MS/animal por dia, respectivamente) em relação aos animais alimentados pelas dietas 1 e 2 (médias de consumo de 549,4 e 696,5 e de produção de fezes de 214,8 e $270,3 \mathrm{~g}$ de MS/animal por dia, respectivamente). Sabe-se que o desempenho animal está diretamente relacionado com o consumo de MS, e as alterações na relação volumoso:concentrado e na qualidade do volumoso podem influenciá-lo (SOEST, 1994). Dessa forma, GASTALDI \& SILVA SOBRINHO (1998) verificaram que ovinos alimentados com dietas contendo 50\% de volumoso ingeriram maior quantidade de MS em relação aos que receberam dieta contendo $70 \%$ de volumoso, em restrição ao concentrado.

Ao se observarem os efeitos das dietas sobre os teores de ST, nota-se que as menores concentrações $(\mathrm{P}<0,05)$ ocorreram nos dejetos de animais alimentados com a dieta $3(28,7 ; 25,9$; 37,4 e $36,8 \%$ de ST nas fezes de animais das categorias 1; 2; 3 e 4, respectivamente), em comparação com as dietas 1 e 2 (médias de 29,3; 32,2; 46,0 e 44,7\% de ST nas fezes das categorias $1 ; 2 ; 3$ e 4 , respectivamente). Os resultados obtidos foram semelhantes aos verificados por AGRAZ (1984), média de $35,4 \%$ de ST.

As cabras da categoria 4 excretaram fezes com maior concentração de $\mathrm{N}(\mathrm{P}<0,05)$, em média $2,15 \%$ da MS (Tabela 1), em relação às categorias 3 e 2, que, por sua vez, foram maiores que a média de excreção da categoria 1 , de $1,0 \% \mathrm{~N}$ da $\mathrm{MS}$. Em relação às diferenças $(\mathrm{P}<0,05)$ atribuídas pelas dietas sob o teor de $\mathrm{N}$ nas fezes, observa-se que os teores excretados por animais que consumiram a dieta 3 foram, superiores aos das dietas 1 e 2 . Na média, a excreção de $\mathrm{N}$ nas fezes de caprinos foi próxima aos dados presentes na literatura, que, segundo AGRAZ (1989), podem oscilar entre 1,7 a 2,0\% da MS para cabras estabuladas, e entre 1,2 e 1,5\% da MS para animais em pastoreio.

Os resultados demonstraram que as categorias de idade dos animais, assim como as dietas oferecidas, afetaram as concentrações de $\mathrm{P}$ e $\mathrm{Mg}$ nas fezes, ou seja, os maiores teores $(\mathrm{P}<0,05)$ excretados foram pela categoria 4, seguida pelas categorias 2 e 3, ficando a categoria $1 \mathrm{com}$ as menores concentrações $(\mathrm{P}<0,05)$. As dietas promoveram elevação, com diferenças significativas, na quantidade excretada desses elementos, de acordo com o aumento de concentrado nas dietas. AGRAZ (1989) observou valores que variaram de 1,2 a 2,5\% de P na MS, no entanto o autor não especificou a idade nem o regime alimentar dos animais. Para o $\mathrm{Mg}$, não foram encontrados dados de excreção para caprinos, no entanto HORN et al. (1994), ao trabalharem com bovinos leiteiros, encontraram resultados de $\mathrm{Mg}$ nas fezes em torno de 0,05 e $0,08 \mathrm{~kg} \mathrm{dia}^{-1}$ que, considerando o teor de ST de aproximadamente $10 \%$ e $88,4 \mathrm{~kg}$ de dejetos por dia, resultaria, em média, $0,6 \%$ de $\mathrm{Mg}$ na MS.

Os animais alimentados com a dieta 3 produziram fezes mais ricas $(\mathrm{P}<0,05)$ em $\mathrm{Ca}$ e $\mathrm{K}$, comparados aos alimentados com a dieta 2, que, por sua vez, tiveram fezes mais concentradas com relação à dieta 1 . Os valores de K podem ser comparados aos obtidos por AGRAZ (1989) em dejetos de caprinos, os quais oscilaram entre 0,1 e $0,6 \%$ na MS. A concentração média de Ca nas fezes (0,9\% na MS) foi semelhante ao encontrado por DORIGAN (2000), 0,8\% de Ca na MS de fezes de caprinos. 
TABELA 1. Teores de macronutrientes, em porcentagem da matéria seca, e micronutrientes, em gramas e $\mathrm{mg} \mathrm{kg}^{-1}$ de matéria seca de fezes, segundo as categorias de idade e dietas administradas.

\begin{tabular}{lcccccccccc}
\hline & $\mathrm{N}$ & $\mathrm{P}$ & $\mathrm{K}$ & $\mathrm{Ca}$ & $\mathrm{Mg}$ & $\mathrm{Fe}$ & $\mathrm{Na}$ & $\mathrm{Zn}$ & $\mathrm{Mn}$ & $\mathrm{Cu}$ \\
\cline { 2 - 11 } & -------------------- & $\% \mathrm{da} \mathrm{MS}$ & ----------------- & $---\mathrm{g} \mathrm{kg}^{-1}$ & $\mathrm{MS}---$ & $--------\mathrm{mg} \mathrm{kg}^{-1} \mathrm{de} \mathrm{MS}--------$ \\
\hline $\mathrm{C} 1 \mathrm{D} 1$ & $0,77 \mathrm{Cb}$ & $0,30 \mathrm{Cc}$ & $0,15 \mathrm{Cc}$ & $0,50 \mathrm{Dc}$ & $0,16 \mathrm{Cc}$ & $0,50 \mathrm{Cb}$ & $0,60 \mathrm{Cc}$ & $78,93 \mathrm{Cc}$ & $162,80 \mathrm{Cc}$ & $13,80 \mathrm{Bc}$ \\
$\mathrm{C} 1 \mathrm{D} 2$ & $0,97 \mathrm{Cb}$ & $0,37 \mathrm{Cb}$ & $0,17 \mathrm{Cb}$ & $0,62 \mathrm{Db}$ & $0,24 \mathrm{Cb}$ & $0,51 \mathrm{Cb}$ & $0,74 \mathrm{Cb}$ & $126,32 \mathrm{Cb}$ & $184,80 \mathrm{Cb}$ & $16,40 \mathrm{Bb}$ \\
$\mathrm{C} 1 \mathrm{D} 3$ & $1,27 \mathrm{Ca}$ & $0,60 \mathrm{Ca}$ & $0,20 \mathrm{Ca}$ & $0,77 \mathrm{Da}$ & $0,32 \mathrm{Ca}$ & $0,70 \mathrm{Ca}$ & $1,02 \mathrm{Ca}$ & $157,17 \mathrm{Ca}$ & $261,80 \mathrm{Ca}$ & $25,53 \mathrm{Ba}$ \\
$\mathrm{C} 2 \mathrm{D} 1$ & $0,90 \mathrm{BCb}$ & $0,44 \mathrm{Bc}$ & $0,16 \mathrm{Cc}$ & $0,70 \mathrm{Cc}$ & $0,28 \mathrm{Bc}$ & $0,52 \mathrm{Bb}$ & $0,76 \mathrm{Bc}$ & $84,45 \mathrm{Cc}$ & $272,80 \mathrm{Cc}$ & $18,67 \mathrm{Bc}$ \\
$\mathrm{C} 2 \mathrm{D} 2$ & $0,98 \mathrm{BCb}$ & $0,55 \mathrm{Bb}$ & $0,19 \mathrm{Cb}$ & $0,79 \mathrm{Cb}$ & $0,34 \mathrm{Bb}$ & $0,58 \mathrm{Bb}$ & $1,05 \mathrm{Bb}$ & $153,50 \mathrm{Cb}$ & $283,80 \mathrm{Cb}$ & $26,93 \mathrm{Bb}$ \\
$\mathrm{C} 2 \mathrm{D} 3$ & $1,59 \mathrm{BCa}$ & $0,61 \mathrm{Ba}$ & $0,21 \mathrm{Ca}$ & $0,89 \mathrm{Ca}$ & $0,38 \mathrm{Ba}$ & $0,76 \mathrm{Ba}$ & $1,25 \mathrm{Ba}$ & $174,87 \mathrm{Ca}$ & $301,18 \mathrm{Ca}$ & $44,33 \mathrm{Ba}$ \\
$\mathrm{C} 3 \mathrm{D} 1$ & $0,99 \mathrm{Bb}$ & $0,52 \mathrm{Bc}$ & $0,24 \mathrm{Bc}$ & $0,96 \mathrm{Bc}$ & $0,40 \mathrm{Bc}$ & $0,56 \mathrm{Bb}$ & $0,80 \mathrm{Bc}$ & $240,27 \mathrm{Bc}$ & $286,95 \mathrm{Bc}$ & $31,33 \mathrm{Ac}$ \\
$\mathrm{C} 3 \mathrm{D} 2$ & $1,06 \mathrm{Bb}$ & $0,63 \mathrm{Bb}$ & $0,30 \mathrm{Bb}$ & $1,01 \mathrm{Bb}$ & $0,45 \mathrm{Bb}$ & $0,60 \mathrm{Bb}$ & $1,03 \mathrm{Bb}$ & $259,60 \mathrm{Bb}$ & $297,00 \mathrm{Bb}$ & $73,80 \mathrm{Ab}$ \\
$\mathrm{C} 3 \mathrm{D} 3$ & $1,71 \mathrm{Ba}$ & $0,72 \mathrm{Ba}$ & $0,45 \mathrm{Ba}$ & $1,10 \mathrm{Ba}$ & $0,53 \mathrm{Ba}$ & $0,83 \mathrm{Ba}$ & $1,32 \mathrm{Ba}$ & $313,80 \mathrm{Ba}$ & $318,15 \mathrm{Ba}$ & $86,60 \mathrm{Aa}$ \\
$\mathrm{C} 4 \mathrm{D} 1$ & $1,69 \mathrm{Ab}$ & $0,80 \mathrm{Ac}$ & $0,36 \mathrm{Ac}$ & $1,17 \mathrm{Ac}$ & $0,61 \mathrm{Ac}$ & $0,73 \mathrm{Ab}$ & $0,98 \mathrm{Ac}$ & $263,13 \mathrm{Ac}$ & $305,60 \mathrm{Ac}$ & $36,87 \mathrm{Ac}$ \\
$\mathrm{C} 4 \mathrm{D} 2$ & $1,84 \mathrm{Ab}$ & $0,89 \mathrm{Ab}$ & $0,46 \mathrm{Ab}$ & $1,27 \mathrm{Ab}$ & $0,68 \mathrm{Ab}$ & $0,79 \mathrm{Ab}$ & $1,34 \mathrm{Ab}$ & $324,13 \mathrm{Ab}$ & $327,60 \mathrm{Ab}$ & $80,93 \mathrm{Ab}$ \\
$\mathrm{C} 4 \mathrm{D} 3$ & $2,93 \mathrm{Aa}$ & $0,97 \mathrm{Aa}$ & $0,53 \mathrm{Aa}$ & $1,54 \mathrm{Aa}$ & $0,78 \mathrm{Aa}$ & $1,01 \mathrm{Aa}$ & $2,13 \mathrm{Aa}$ & $389,47 \mathrm{Aa}$ & $352,00 \mathrm{Aa}$ & $94,40 \mathrm{Aa}$ \\
\hline Média & 1,39 & 0,62 & 0,29 & 0,94 & 0,43 & 0,67 & 1,09 & 205,47 & 280,14 & 45,80 \\
\hline C.V. (\%) & 21,89 & 23,36 & 25,57 & 24,42 & 24,91 & 22,9 & 21,75 & 36,84 & 27,46 & 79,48 \\
\hline
\end{tabular}

C1 - categoria 1; C2 - categoria 2; C3 - categoria 3; C4 - categoria 4; D1 - dieta 1; D2 - dieta 2, e D3 - dieta 3.

Médias seguidas de letras diferentes maiúsculas na coluna diferem entre si, pelo teste de Tukey $(\mathrm{P}<0,05)$, e comparam categorias.

Médias seguidas de letras diferentes minúsculas na coluna diferem entre si, pelo teste de Tukey $(\mathrm{P}<0,05)$, e comparam dietas.

A quantidade de $\mathrm{N}$ excretada na urina (Tabela 2) foi maior $(\mathrm{P}<0,05)$ em animais adultos, comparada à excreção dos animais da categoria 3, que, por sua vez, superou as categorias 1 e 2 . Os resultados obtidos foram inferiores aos preconizados por AGRAZ (1984), no mínimo 10,0 g de $\mathrm{N}$ por dia na urina, no entanto o referido autor não mencionou o regime alimentar nem a idade dos animais. SOEST (1994) ressaltou que a elevação de alimento concentrado na dieta aumenta a quantidade de proteína rapidamente degradável e, caso essa quantidade seja superior à captação realizada pelos microrganismos do rúmen, haverá perda de $\mathrm{N}$, na forma de amônia, que será encaminhada ao fígado, transformando-se em uréia, e então liberada na urina.

TABELA 2. Excreção diária (por animal) de macro e micronutrientes na urina de caprinos, de acordo com o volume total produzido, segundo as categorias de idade e dietas administradas.

\begin{tabular}{|c|c|c|c|c|c|c|c|c|c|}
\hline & $\mathrm{N}$ & $\mathrm{P}$ & $\mathrm{K}$ & $\mathrm{Na}$ & $\mathrm{Ca}$ & $\mathrm{Mg}$ & $\mathrm{Fe}$ & $\mathrm{Zn}$ & $\mathrm{Mn}$ \\
\hline & \multicolumn{4}{|c|}{---------------- g dia } & \multicolumn{3}{|c|}{------------ $\mathrm{mg} \mathrm{dia}{ }^{-1}$------------ } & \multicolumn{2}{|c|}{------ $\mu \mathrm{g} \mathrm{dia}^{-1}-----$} \\
\hline C1D1 & $2,67 \mathrm{Cb}$ & $0,32 \mathrm{Cb}$ & $0,45 \mathrm{Bb}$ & $0,24 \mathrm{Bb}$ & $66,50 \mathrm{Dc}$ & $55,11 \mathrm{Bb}$ & $1,60 \mathrm{Cc}$ & $65,41 \mathrm{Dc}$ & $26,82 \mathrm{Cb}$ \\
\hline C1D2 & $21 \mathrm{Cb}$ & $0,33 \mathrm{Cb}$ & $0,48 \mathrm{Bb}$ & $26 \mathrm{Bb}$ & $79,20 \mathrm{Db}$ & $66,20 \mathrm{Bab}$ & $2,23 \mathrm{Cb}$ & $73,81 \mathrm{Db}$ & $31,12 \mathrm{Cb}$ \\
\hline ID3 & $46 \mathrm{Ca}$ & $0,56 \mathrm{Ca}$ & $0,63 \mathrm{Ba}$ & $34 \mathrm{Ba}$ & 4,60Da & $74,58 \mathrm{Ba}$ & $2,46 \mathrm{Ca}$ & $86,88 \mathrm{Da}$ & $35,96 \mathrm{Ca}$ \\
\hline & $15 \mathrm{Cb}$ & $0,38 \mathrm{Cb}$ & $0,46 \mathrm{Bb}$ & $0,23 \mathrm{Bb}$ & $81,90 \mathrm{Cc}$ & $65,69 \mathrm{Bb}$ & $1,64 \mathrm{Cc}$ & $74,62 \mathrm{Cc}$ & $31,63 \mathrm{Cb}$ \\
\hline & $77 \mathrm{Cb}$ & $0,35 \mathrm{Cb}$ & $0,51 \mathrm{Bb}$ & $0,22 \mathrm{Bb}$ & $96,20 \mathrm{Cb}$ & $77,84 \mathrm{~B}$ & $2,12 \mathrm{Cb}$ & $84,13 \mathrm{Cb}$ & $45,56 \mathrm{Cb}$ \\
\hline & $1 \mathrm{Ca}$ & $0,57 \mathrm{Ca}$ & $0,65 \mathrm{Ba}$ & $0,36 \mathrm{Ba}$ & $114,40 \mathrm{Ca}$ & $87,39 \mathrm{Ba}$ & $2,38 \mathrm{Ca}$ & $97,45 \mathrm{Ca}$ & $60,45 \mathrm{Ca}$ \\
\hline e & $61 \mathrm{Bb}$ & $0,48 \mathrm{Bb}$ & $0,57 \mathrm{ABb}$ & $0,29 \mathrm{Ab}$ & $101,40 \mathrm{Bc}$ & $78,32 \mathrm{ABb}$ & $2,08 \mathrm{Bc}$ & $89,49 \mathrm{Bc}$ & $64,43 \mathrm{Bb}$ \\
\hline C3D2 & $37 \mathrm{Bb}$ & $0,53 \mathrm{Bb}$ & $0,57 \mathrm{ABb}$ & $0,31 \mathrm{Ab}$ & $112,10 \mathrm{Bb}$ & $89,06 \mathrm{ABab}$ & $2,56 \mathrm{Bb}$ & $98,20 \mathrm{Bb}$ & $72,87 \mathrm{Bb}$ \\
\hline C3D3 & $97 \mathrm{Ba}$ & $0,67 \mathrm{Ba}$ & $0,71 \mathrm{ABa}$ & $0,40 \mathrm{Aa}$ & $128,90 \mathrm{Ba}$ & $97,32 \mathrm{ABa}$ & $2,81 \mathrm{Ba}$ & $105,18 \mathrm{Ba}$ & $88,39 \mathrm{Ba}$ \\
\hline 4D1 & $6,95 \mathrm{Ab}$ & $0,56 \mathrm{Ab}$ & $0,66 \mathrm{Ab}$ & $0,34 \mathrm{Ab}$ & $113,10 \mathrm{Ac}$ & $92,01 \mathrm{Ab}$ & $2,51 \mathrm{Ac}$ & $109,54 \mathrm{Ac}$ & $77,01 \mathrm{Ab}$ \\
\hline & $4 \mathrm{Ab}$ & $0,63 \mathrm{Ab}$ & $8 \mathrm{Ab}$ & $38 \mathrm{Ab}$ &, $50 \mathrm{Ab}$ & $2 \mathrm{Aab}$ & $2,93 \mathrm{Ab}$ & $117,82 \mathrm{Ab}$ & $80,03 \mathrm{Ab}$ \\
\hline C4I & $83 \mathrm{Aa}$ & $0,77 \mathrm{Aa}$ & $0,86 \mathrm{Aa}$ & $0,50 \mathrm{Aa}$ & $145,40 \mathrm{Aa}$ & $116,02 \mathrm{Aa}$ & $3,22 \mathrm{Aa}$ & $129,99 \mathrm{Aa}$ & $96,94 \mathrm{Aa}$ \\
\hline & & & 0,60 & 0,32 & & & 2,38 & 94,37 & 59,24 \\
\hline C.V. $(\%)$ & 39,26 & 69,29 & 33,14 & 76,4 & 34,57 & 62,99 & 48,99 & 72,58 & 32,6 \\
\hline
\end{tabular}

C1 - categoria 1; C2 - categoria 2; C3 - categoria 3; C4 - categoria 4; D1 - dieta 1; D2 - dieta 2 e D3 - dieta 3.

Médias seguidas de letras diferentes maiúsculas na coluna diferem entre si, pelo teste de Tukey $(\mathrm{P}<0,05)$, e comparam categorias.

Médias seguidas de letras diferentes minúsculas na coluna diferem entre si, pelo teste de Tukey $(\mathrm{P}<0,05)$, e comparam dietas. 
A quantidade de $\mathrm{P}$ eliminada na urina foi maior $(\mathrm{P}<0,05)$ em cabras alimentadas pela dieta 3 , em média 0,6 g de $\mathrm{P}$ por dia, em comparação com as dietas 1 e 2, com médias de 0,4 e 0,5 g de $\mathrm{P}$ por dia, respectivamente. Provavelmente, esses resultados são reflexos do consumo das dietas. A excreção de P observada por AGRAZ (1984) foi de $0,7 \mathrm{~g}$ de $\mathrm{P}$ por dia na urina.

Nota-se que os animais alimentados com a dieta 3 geraram urina com maior concentração de $\mathrm{K}$; esse fato pode estar estritamente associado à elevação de concentrado na dieta que, além de contribuir com maiores quantidades de $\mathrm{K}$, elevou o consumo diário.

\section{Biodigestão Anaeróbia}

\section{Redução de SV}

Os resultados apresentaram diferenças $(\mathrm{P}<0,01)$ entre as reduções ocorridas nos teores de $\mathrm{SV}$, desde o início até o final do processo (Tabela 3), quando se compararam os três tipos de substratos, demonstrando que o aumento na quantidade de concentrado na ração permitiu substratos com maior degradabilidade. Ao se compararem as médias de redução de SV, observa-se que animais alimentados com maior quantidade de concentrado (dieta 3) produziram dejetos que, nos biodigestores, apresentaram $45 \%$ de redução nos teores de SV, valor superior aos obtidos com o aumento nos teores de volumoso (35\% para a dieta 2 e $28,6 \%$ para a dieta 1 ).

TABELA 3. Teores de sólidos totais e voláteis, em percentagem e em massa, e redução de SV, em percentagem, para estrumes obtidos com diferentes tipos de dieta dos animais.

\begin{tabular}{|c|c|c|c|c|c|c|c|c|c|}
\hline \multirow{3}{*}{ Dieta } & \multicolumn{4}{|c|}{$\mathrm{ST}$} & \multicolumn{4}{|c|}{$\mathrm{SV}$} & \multirow{3}{*}{$\begin{array}{l}\text { Redução } \\
\text { de SV (\%) }\end{array}$} \\
\hline & \multicolumn{2}{|c|}{$\%$} & \multicolumn{2}{|c|}{$\mathrm{kg}$} & \multicolumn{2}{|c|}{$\%$} & \multicolumn{2}{|c|}{$\mathrm{kg}$} & \\
\hline & inicial & final & inicial & final & inicial & final & inicial & final & \\
\hline 1 & 7,41 & 5,34 & 0,296 & 0,214 & 6,38 & 4,56 & 0,255 & 0,182 & $28,60 \mathrm{C}$ \\
\hline 2 & 7,32 & 5,09 & 0,293 & 0,204 & 6,43 & 4,18 & 0,257 & 0,167 & $34,99 \mathrm{~B}$ \\
\hline 3 & 7,72 & 4,57 & 0,309 & 0,183 & 6,76 & 3,72 & 0,270 & 0,149 & $45,00 \mathrm{~A}$ \\
\hline
\end{tabular}

Médias seguidas de letra distinta, na coluna, diferem pelo teste de Tukey, a 5\%.

AL-MASRI (2001) obteve valores superiores para a redução de SV $(58,1 \%)$ ao utilizar dejetos de caprinos como componente dos substratos, no entanto o autor manteve os biodigestores na temperatura de $30^{\circ} \mathrm{C}$, o que pode ter maximizado a degradação da fração volátil. AMORIM et al. (2004) obtiveram resultados semelhantes ao efetuarem a biodigestão anaeróbia dos dejetos de cabras Saanen adultas durante as quatro estações do ano, obtendo média de $36 \%$ de redução de SV, sem observar efeito $(\mathrm{P}>0,05)$ das estações do ano.

Considerando os resultados obtidos (Figura 1), verificou-se que os substratos preparados com dejetos da dieta 2 permitiram maior velocidade inicial na produção de biogás, embora produzindo menor quantidade $(\mathrm{P}<0,05)$ em relação aos substratos preparados com a dieta 3 (dieta $3=$ $0,072 \mathrm{~m}^{3}$, dieta $2=0,067 \mathrm{~m}^{3}$, dieta $1=0,062 \mathrm{~m}^{3}$ ). Os resultados evidenciaram que as dietas oferecidas aos animais alteraram $(\mathrm{P}<0,05)$ a produção de biogás. Esse fato está associado à produção de fezes com maior concentração de nutrientes, conforme se verificaram nos resultados apresentados nas Tabelas 3 e 4, além de essas fezes conterem maiores quantidades de carbono mais assimiláveis pelos microrganismos, já que os animais foram alimentados com maiores quantidades de concentrado, em restrição ao volumoso.

Considerando as produções de biogás por kg de substrato, por kg de ST adicionados e por kg de SV adicionados (Tabela 4), observa-se que os substratos preparados com dejetos obtidos de animais alimentados com a dieta 3 produziram mais biogás $(\mathrm{P}<0,01)$, indicando que o aumento no concentrado da ração aumentou o potencial energético expresso em produção de biogás. $\mathrm{O}$ maior potencial $(\mathrm{P}>0,05)$ alcançado em biodigestores abastecidos com dejetos da dieta $1, \mathrm{em} \mathrm{m}^{3} \mathrm{de}$ biogás por $\mathrm{kg}$ de $\mathrm{SV}$ reduzidos, pode ser atribuído ao período de retenção do material no biodigestor, que, provavelmente, favoreceu a degradação de compostos mais resistentes, como os componentes da parede celular. 


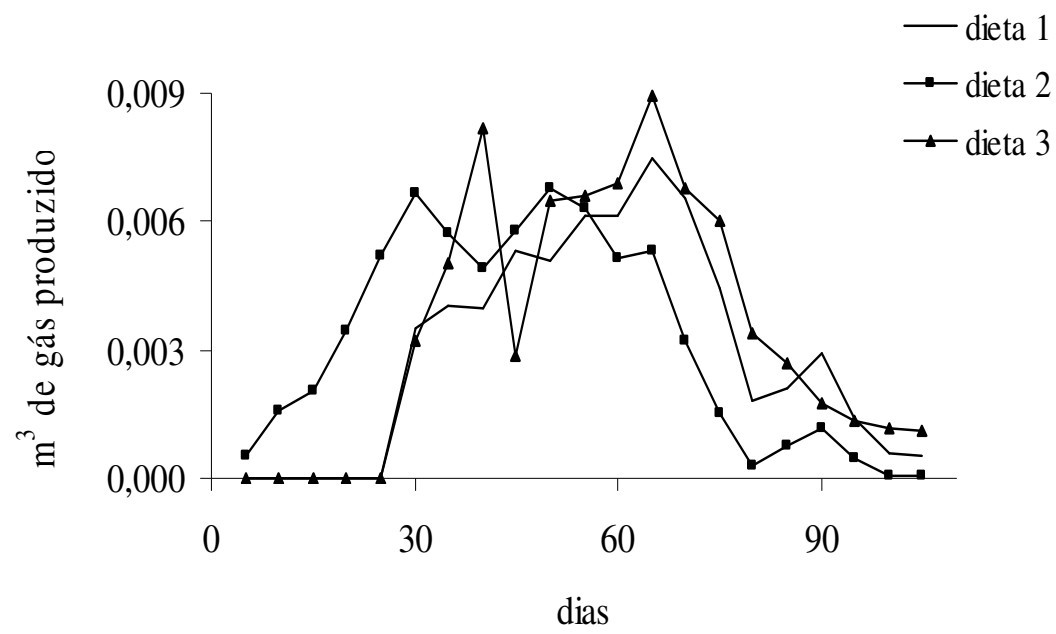

FIGURA 1. Distribuição da produção $\left(\mathrm{m}^{3}\right)$ de biogás, em biodigestores abastecidos com dejetos de caprinos alimentados com dietas variando as proporções entre volumoso e concentrado.

TABELA 4. Potenciais médios de produção de biogás, corrigidos para $20{ }^{\circ} \mathrm{C}$ e $1 \mathrm{~atm}$, para os diferentes tipos de substratos, preparados com dejetos de animais alimentando-se com três dietas.

\begin{tabular}{cccccc}
\hline \multirow{2}{*}{ Dieta } & \multicolumn{5}{c}{ Potenciais $\left(\mathrm{m}^{3} \mathrm{~kg}^{-1}\right)$} \\
\cline { 2 - 6 } & Substrato & ST Adicionado SV Adicionado & SV Reduzido & Dejeto \\
\hline 1 & $0,0155 \mathrm{~B}$ & $0,2097 \mathrm{~B}$ & $0,2434 \mathrm{~B}$ & $0,8503 \mathrm{~A}$ & $0,0327 \mathrm{~A}$ \\
2 & $0,0168 \mathrm{~B}$ & $0,2289 \mathrm{AB}$ & $0,2610 \mathrm{AB}$ & $0,7453 \mathrm{~B}$ & $0,0319 \mathrm{~A}$ \\
3 & $0,0181 \mathrm{~A}$ & $0,2341 \mathrm{~A}$ & $0,2680 \mathrm{~A}$ & $0,5979 \mathrm{C}$ & $0,0302 \mathrm{~B}$ \\
\hline
\end{tabular}

Nas colunas, médias seguidas de letras distintas diferem pelo teste de Tukey, a 5\%.

O melhor parâmetro para refletir o potencial de determinada biomassa e, portanto, mais indicado para se utilizar em projetos de biodigestores, é aquele que expressa a produção de biogás por $\mathrm{kg}$ de ST adicionados nos biodigestores, pois elimina a interferência do teor de água presente na biomassa. No que se relaciona aos potenciais expressos dessa maneira, observa-se que a dieta 3 pode aumentar a produção de biogás $(\mathrm{P}<0,01)$ em aproximadamente $10,5 \%$ em relação à dieta 1 .

AMORIM et al. (2004) verificaram maiores potenciais de produção de biogás por $\mathrm{kg}$ de substrato e $\mathrm{kg}$ de dejetos adicionados aos biodigestores (médias de 0,02 e 0,05 $\mathrm{m}^{3}$ de biogás, respectivamente), ao utilizarem os dejetos de cabras Saanen adultas como substratos para a biodigestão anaeróbia, nas quatro estações do ano. Os maiores potenciais obtidos pelos referidos autores podem ser atribuídos à maior capacidade dos biodigestores utilizados $(60 \mathrm{~L})$ e à condição de manutenção desses (em campo e aterrados no solo), além das diferenças nas características dos dejetos que podem estar relacionadas com a alimentação dos animais, já que, no referido estudo, foi ofertado às cabras silagem de milho e concentrado, na proporção 50:50. Esse último fator pode ter sido a principal causa dos maiores potenciais observados pelos autores, já que a silagem de milho se constitui em volumoso de melhor qualidade que o feno, principalmente pelos menores conteúdos de parede celular, o que limita a degradação do alimento (no animal) e dos dejetos (nos biodigestores).

MISI \& FORSTER (2001) efetuaram a biodigestão anaeróbia de misturas à base de dejetos de caprinos, ovinos e bovinos, resíduos do processamento de frutas e cama de frangos, em diferentes proporções, e observaram que, quando os dejetos de ovinos e caprinos perfizeram $70 \%$ das misturas, obtiveram como valores: $0,14 \mathrm{~m}^{3}$ de metano por $\mathrm{kg}$ de $\mathrm{SV}$ adicionado e $0,42 \mathrm{~m}^{3} \mathrm{de}$ $\mathrm{CH}_{4}$ por $\mathrm{kg}$ de $\mathrm{SV}$. Valores superiores de potenciais de produção de biogás foram observados por JAIN et al. (1981) e LUCAS JÚNIOR (1987) ao efetuarem a biodigestão anaeróbia dos dejetos de 
ovinos e bovinos $\left(0,045\right.$ e $0,048 \mathrm{~m}^{3}$ de biogás por $\mathrm{kg}$ de dejeto), respectivamente. Esses autores mencionados não relataram a alimentação dos animais, fato que pode ter ocasionado as diferenças entre os resultados de potenciais de produção de biogás que foram obtidos e os presentes na literatura.

\section{CONCLUSÕES}

As características quantitativas e qualitativas dos dejetos excretados por caprinos variaram com a faixa etária em que os animais se encontraram, assim como sofreram interferência do tipo de alimentação que lhes foi oferecida. As concentrações de nutrientes contidas nos dejetos gerados por cabras foram maiores em animais adultos e alimentados com dietas mais ricas em concentrado.

As maiores proporções de concentrado na dieta dos caprinos proporcionaram substratos que, durante a biodigestão anaeróbia, propiciaram maiores reduções de SV e produções de biogás.

\section{REFERÊNCIAS}

AGRAZ G.A.A. Caprinotecnia 1. México: Limusa, 1984. 829 p.

AGRAZ G.A.A. Caprinotecnia 2. $2^{\text {nd }}$ ed. México: Limusa, 1989. 1.212 p.

AL-MASRI, M.R. Changes in biogas production due to different ratios of some animal and agricultural wastes. Bioresource Technology, Oxford, v.77, n.1, p.97-100, 2001.

AMORIM, A.C.; LUCAS JÚNIOR, J.; RESENDE, K.T. Efeito da estação do ano sobre a biodigestão anaeróbia de dejetos de caprinos. Engenharia Agrícola, Jaboticabal, v.24, n.1, p.16-24, 2004.

AMERICAN PUBLIC HEALTH ASSOCIATION. Standard methods for the examination of water and wastewater. $19^{\text {th }}$ ed. Washington, 1995. "pg. irreg."

AUGENSTEIN, D.; BENEMANN, J.; HUGHES, E. Electricity from biogas. In: National Bioenergy Conference, 6., 1994, Nevada. Anais... Reno - Sparks: Judy Farell, 1994. p.1.237-40.

CAETANO, L. Proposição de um sistema modificado para quantificação de biogás. 1985. 75 f.

Dissertação (Mestrado em Energia na Agricultura) - Faculdade de Ciências Agronômicas, Universidade Estadual Paulista, Botucatu, 1985.

DORIGAN, C.J. Metabolismo e perdas endógenas de cálcio em cabritos Saanen. 2000. 114 f. Tese. (Doutorado em Produção Animal) - Faculdade de Ciências Agrárias e Veterinárias, Universidade Estadual Paulista, Jaboticabal, 2000.

DUBEUF, J.P.; MORAND-FEHR, P.; RUBINO, R. Situation, changes and future of goat industry around the world. Small Ruminant Research, Thessaloniki, v.51, n.2, p.165-73, 2004.

EL AICH, A.; WATERHOUSE, A. Small ruminants in environmental conservation. Small Ruminant Research, Thessaloniki, v.34, n.3, p.271-87, 1999.

GASTALDI, K.A.; SILVA SOBRINHO, A.G. Desempenho de ovinos F1 Ideal x Ile de France em confinamento com diferentes relações concentrado: volumoso. In: REUNIÃO ANUAL DA SOCIEDADE BRASILEIRA DE ZOOTECNIA, 35., 1998, Botucatu. Anais... 1 CD-ROM.

HORN, H.H.Van; WILKIE, A.C.; POWERS, W.J.; NORDSTEDT, R.A. Components of dairy manure management systems. Journal Dairy Science, Savoy, v.77, n.7, p.2008-30, 1994.

JAIN, M.K.; SINGH, R.; TAURO, P. Anaerobic digestion of cattle and sheep wastes. Agricultural Wastes, London, v.3. n.3, p.91-8, 1981.

JARDIM, W.R. Criação de caprinos. 3.ed. São Paulo: Nobel, 1977. 239 p.

LUCAS JÚNIOR, J. Algumas considerações sobre o uso do estrume de suínos como substrato para três sistemas de biodigestores anaeróbios. 1994. 137 f. Tese (Livre-Docência em Construções 
Rurais) - Faculdade de Ciências Agrárias e Veterinárias, Universidade Estadual Paulista, Jaboticabal, 1994.

LUCAS JÚNIOR, J. Estudo comparativo de biodigestores modelo indiano e chinês. 1987. $114 \mathrm{f}$. Tese (Doutorado em Energia na Agricultura) - Faculdade de Ciências Agronômicas, Universidade Estadual Paulista, Botucatu, 1987.

MISI, S.N.; FORSTER, C.F. Batch co-digestion of multi-componente agro-wastes. Bioresource Technology, Oxford, v.80, n.1, p.19-28, 2001.

NRC. NUTRITION REQUERIMENTS OF GOATS. Washington: National Academy Press, 1981. p.2-3.

RIBEIRO, S.D.A.; RIBEIRO, A.C. Produção de carne caprina: situação atual e perspectivas. In: REUNIÃO ANUAL DA SOCIEDADE BRASILEIRA DE ZOOTECNIA, 38., 2001, Botucatu. Anais... 2001. 1 CD-ROM.

SAHA, S.L. Promoting use of the biogas in India. Electrical India, Calcutta, v.34, n.1, p.13-16, 1994.

SAS. SAS/STAT - User's Guide. Version 6. $4^{\text {th }}$. ed. Cary, 1990. 891 p.

SOEST, P.J. Van. Nutritional ecology of the ruminant. $2^{\text {nd }}$ ed. Ithaca: Cornell University, 1994. $476 \mathrm{p}$. 